\title{
Kapitalismus oder Solidarität?* \\ Zur Bedeutung der Grundlagen der Sozialen Marktwirtschaft angesichts der aktuellen Infragestellung marktwirtschaftlicher Funktionsprinzipien
}

\author{
GEORg TRAUTNITZ ${ }^{* *}$
}

\section{Capitalism or Solidarity? About the Relevance of the Foundations of the Social Market Economy in View of the Current Critique of the Principles of a Market Economy}

In view of the current critique of capitalism this paper tries to ground the concept of the Social Market Economy in the philosophy of ethics and economics. The reflection on the conditions of the possibility of economic exchange shows that the social element in the Social Market Economy is not an arbitrary modification of the "natural" market order. Instead the paper gives evidence that individual freedom for more than one person cannot be conceived of without acknowledging the intersubjective genesis of individual freedom. This intersubjective genesis bounds the economic and the social perspective inextricably together. By illuminating this interconnectedness the paper finally rejects the current calls for a fundamental reform of the market economy as unjustified.

Keywords: Ethics and Economics, Pbilosophical Foundations of the Social Market Economy, Market Order, Constitutional Economics

\section{Die Finanz- und Wirtschaftskrise als Ausgangspunkt für eine grundsätz- liche Kapitalismuskritik}

Die Finanz- und Wirtschaftskrise von 2007 bis 2009 und die Maßnahmen zu ihrer Bekämpfung wurden von verschiedensten Seiten zum Anlass genommen, um die Funktionsprinzipien kapitalistischer Wirtschaftssysteme grundsätzlich infrage zu stellen. ${ }^{1}$

Insbesondere der Finanzsektor hat aufgrund seiner Kreditverbriefungs-Industrie die altbekannten Vorwürfe von,Gewinnsucht' und ,Gier' provoziert. Diese Vorwürfe enthalten dann eine besondere gesellschaftskritische Schärfe, wenn sie nicht als moralische Diskreditierung besonders krasser Fehlleistungen einzelner Akteure formuliert werden, sondern als analytische Beschreibung eines systemischen Merkmals.

Der langjährige Richter am Bundesverfassungsgericht Ernst-Wolfgang Böckenförde hat die letzte Finanz- und Wirtschaftskrise in diesem Sinne zum Anlass genommen,

\footnotetext{
* Beitrag eingereicht am 05.03.2010, nach doppelt verdecktem Gutachterverfahren überarbeitete Fassung angenommen am 10.01.2011.

** Dr. Georg Trautnitz, Wissenschaftlicher Mitarbeiter, Lehrstuhl für Internationales Management, Otto-Friedrich-Universität Bamberg, Kirschäckerstr. 39, D-96045 Bamberg, Tel.: +49-(0)9518632672, Fax: +49-(0)951-8632667, E-Mail: georg.trautnitz@uni-bamberg.de, Forschungsschwerpunkte: Wirtschaftsethik, Rechts- und Moralphilosophie, Internationales Management, Theorie der Unternehmung, Corporate Governance.

1 Vgl. beispielhaft: Dörre et al. 2010; Schallmayer 2009; Trojanow 2009.
} 
die Grundlagen marktwirtschaftlicher Wirtschaftssysteme grundsätzlich zu kritisieren (vgl. dazu und im Folgenden: Böckenförde 2009). Nach Böckenförde zeigt diese Krise den ,inhumanen Charakter" des kapitalistischen Wirtschaftssystems, das nicht länger lediglich in seinen Symptomen und Nebenwirkungen abgemildert werden darf, vielmehr einer grundlegenden Systemkritik zu unterziehen ist, die auf ein „Gegenmodell zum Kapitalismus“ hinausläuft. Böckenförde hält den Ausgangspunkt des „Kapitalismus" für prinzipiell verfehlt und fordert im Anschluss an die Tradition der katholischen Soziallehre die „Umkehrung“ dieses Ausgangspunktes:

\begin{abstract}
„An die Stelle eines ausgreifenden Besitzindividualismus, der das als natürliches Recht proklamierte potentiell unbegrenzte Erwerbsinteresse der Einzelnen, das keiner inhaltlichen Orientierung unterliegt, zum Ausgangspunkt und strukturierenden Prinzip nimmt, müssen ein Ordnungsrahmen und eine Handlungsstrategie treten, die davon ausgehen, dass die Güter der Erde [...] nicht denjenigen gehören, die sie sich zuerst aneignen und ausnützen, sondern zunächst allen Menschen gewidmet sind, zur Befriedigung ihrer Lebensbedürfnisse und der Erlangung von Wohlfahrt“" (Böckenförde 2009).
\end{abstract}

Am Ende der Ausführungen von Böckenförde wird deutlich, dass er mit diesen Worten nicht etwa eine spezifische - meist als anglo-amerikanisch bezeichnete - Form des Kapitalismus kritisieren möchte, gegen die er die Grundlagen einer sozialen Marktwirtschaft profiliert. Vielmehr zielt er auf einen grundsätzlichen Umbau der kapitalistischen Wirtschaftsordnung auch in Europa und damit eben auch in Deutschland.

Inhaltlich möchte Böckenförde das marktwirtschaftliche System von einem Besitzindividualismus umstellen auf eine solidarische Orientierung. Er hält mit Thomas von Aquin die Institution des Privateigentums für eine derivative Größe, die sich aus lediglich pragmatischen Gründen aus der gottgegebenen Widmung der irdischen Güter ergibt. Wenn diese Güter „daraufhin geordnet sind, der Bedürftigkeit der Menschen insgesamt aufzuhelfen", dann ergibt sich das Privateigentum lediglich aus Gründen der Zweckmäßigkeit. Es gehört nicht zum sakrosankten Kern der natürlichen bzw. von Gott gegebenen Ordnung.

Mit dem Besitzindividualismus stellt Böckenförde in der Tat die zentrale Basis des marktwirtschaftlichen Wirtschaftssystems in Frage. Falls die gegenwärtige marktwirtschaftliche Strukturierung unseres Wirtschaftssystems tatsächlich als prinzipiell ungerecht zu verwerfen wäre, ergäbe sich aus dieser Einsicht ein enormes gesellschaftspolitisches Konfliktpotential. ${ }^{2}$ Wer die bestehenden Verhältnisse für grundlegend ungerecht hält, der sieht sich - je nach ideologischer Ausrichtung - einer geschichtlichen, moralischen und/oder religiösen Verpflichtung ausgesetzt, diese Verhältnisse zu bekämpfen. Jedenfalls zeigt die Geschichte des vergangenen Jahrhunderts, welche verheerenden Folgen ein grundlegender gesellschaftlicher Dissens in der Systemfrage für das menschliche Zusammenleben haben kann.

Die Kritik Böckenfördes am systemischen Charakter des Kapitalismus ist so grundlegend, dass sie als Wiederaufgreifen der Systemfrage aufgefasst werden kann. Der fol-

2 Zur Bedeutung eines allgemeinen gesellschaftlichen Konsenses über die Grundprinzipien der Sozialen Marktwirtschaft siehe Schlecht 1999: 296 f. 
gende Beitrag konzentriert sich deshalb auf die Entkräftung dieser Fundamentalkritik, ${ }^{3}$ ohne auf die Verzweigungen der aktuellen Kapitalismuskritik insgesamt einzugehen. In diesem Beitrag soll es ausschließlich um die Frage gehen, ob die Grundidee der Sozialen Marktwirtschaft unter einem wirtschaftsethischen Anspruch in einer Weise gerechtfertigt werden kann, die die Systemfrage obsolet werden lässt.

Der hier veranschlagte Rechtfertigungsbegriff beschränkt sich nicht auf eine bloße Apologetik der Sozialen Marktwirtschaft. Es soll also nicht darum gehen, Wertvorstellungen zu suchen, die den Gestaltungsprinzipien der Sozialen Marktwirtschaft zugrunde liegen (könnten). Ein solches apologetisches Verfahren erschöpfte sich darin zu zeigen, dass es nicht unvernünftig sein muss, die Grundideen der Sozialen Marktwirtschaft als Gestaltungsprinzipien des Wirtschaftssystems zu etablieren. Es bliebe aber offen, ob es nicht doch auch andere, eventuell sogar validere Wertvorstellungen geben könnte, von denen ausgehend sich alternative Systementwürfe ableiten bzw. rechtfertigen ließen.

Zur Überwindung einer solchen Apologetik ist es erforderlich, die Grundidee der Sozialen Marktwirtschaft aus einem Prinzip abzuleiten, das mit dem lediglich formalen Gedanken des Zusammenlebens von Menschen verbunden ist. Es ginge also nicht mehr um die Frage, unter welchen Wertgesichtspunkten diese Menschen ihr Zusammenleben in bestimmten Hinsichten gestalten wollen. Es ginge nicht mehr um die Gestaltung des sozialen und wirtschaftlichen Lebens innerhalb einer vorausgesetzten Grundordnung. Nicht die Frage nach dem, guten Leben' wäre zu stellen, sondern die Frage nach den Bedingungen, die ein Zusammenleben von Menschen überhaupt erst ermöglichen.

Sollte die Soziale Marktwirtschaft aus einem solchen Prinzip abgeleitet werden können, dann ließe sich einsichtig machen, dass sie kein beliebiges ökonomisches und politisches Modell ist, für dessen Rechtfertigung sich zwar gewichtige Gründe anführen lassen, das letztlich aber auf kontingenten (Wert-)Annahmen beruht. Vielmehr würde die Ableitung aus dem rein formalen Gesichtspunkt des Zusammenlebens von Menschen der Sozialen Marktwirtschaft einen prinzipiellen Geltungsanspruch sichern.

Im folgenden Abschnitt wird ein kurzer Abriss der ideengeschichtlichen Grundlagen der Sozialen Marktwirtschaft gegeben, der zeigt, worin die konzeptionelle Schwäche dieser Grundlagen besteht. Abschnitt drei versucht, der Sozialen Marktwirtschaft nachträglich ein neues Begründungsfundament zu geben, das diese Schwäche vermeidet. Der letzte Abschnitt greift die Systemkritik Böckenfördes auf und diskutiert, inwiefern sich ihr Anliegen auch auf Grundlage des zuvor dargestellten Begründungsfundamentes der Sozialen Marktwirtschaft umsetzen lässt.

\section{Die unklaren Grundlagen der Sozialen Marktwirtschaft}

Die aktuelle Kapitalismuskritik deckt nochmals deutlich die schon vieldiskutierte konzeptionelle Schwäche der Sozialen Marktwirtschaft auf (vgl. Ptak 2004: 295ff.): Die mangelnde Genauigkeit in der Bestimmung des Verhältnisses zwischen dem markt-

3 Im Rahmen dieses Beitrages kann die differenzierte rechts- und staatsphilosophische Position Böckenfördes nicht berücksichtigt werden. Der Beitrag bezieht sich ausschließlich auf die von Böckenförde kürzlich vorgetragene Kapitalismuskritik. 
wirtschaftlichen und dem sozialen Element. Die intellektuellen Strömungen und konzeptionellen Traditionen, durch deren Kombination und Zusammenschau die politische Leitidee der Sozialen Marktwirtschaft entstanden ist, bestimmen dieses Verhältnis in unterschiedlicher Weise. Die vielfältigen gedanklichen und auch persönlichen Verbindungslinien zwischen den Personengruppen, die die Idee der Sozialen Marktwirtschaft entscheidend beeinflusst haben, sowie die von ihnen vorgenommenen Nuancierungen und Interpretationen dieser Idee können hier nicht dargestellt werden. ${ }^{4}$ Bezüglich der Frage nach dem Verhältnis zwischen dem marktwirtschaftlichen und dem sozialen Element in der Sozialen Marktwirtschaft lassen sich jedoch vereinfachend zwei Traditionsstränge gegenüberstellen.

Für Gustav von Schmollers (1838-1917) Theorie der ökonomischen Entwicklung ist der Gedanke der gesamtheitlichen Gestalt jeder historisch nachweisbaren, Volkswirtschaft ${ }^{\natural}$ von besonderer Bedeutung. Demnach ist für das verstehende Betrachten wirtschaftlicher Zusammenhänge deren Verortung in übergreifenden sozialen und psychologischen Verhaltensweisen unerlässlich (vgl. Schmoller 1898: 220f.). Daraus folgt die Betonung der Notwendigkeit, die ökonomische Wissenschaft auf Grundlage von Beobachtungen und Beschreibungen zu entwickeln, die die Besonderheiten der historisch vorliegenden wirtschaftlichen Gebilde abbilden, um daran anknüpfend eventuelle (Verhaltens-)Muster und Regelmäßigkeiten aufdecken zu können (vgl. Schmoller 1901: 100ff., insbes. 110). ${ }^{5}$ Dieser konzeptionellen Einbettung des Ökonomischen in übergeordnete soziale Zusammenhänge entspricht auch die Gegenüberstellung von zwei Grundkräften der historischen Entwicklung von Gesellschaften (vgl. Schmoller 1898: 126): Einerseits des Wettbewerbs mit seiner „natürlichen“ Selektionsfunktion der Tüchtigsten und andererseits der Kooperation mit ihrer ethisch-kulturellen Basis. Aus der Annahme einer grundsätzlichen Spannung zwischen beiden Kräften ergibt sich unmittelbar Schmollers Forderung nach einer „Socialpolitik“. Diese soll die sich zwangsläufig aus der Entwicklungslogik des Wettbewerbs ergebenden sozialen Verwerfungen abmildern sowie die rein materielle Verbesserung der Lebensumstände durch die Förderung einer kulturellen Entwicklung ergänzen (vgl. Schmoller 1918: 333f.). Dass die naturwüchsige Dynamik der ökonomisch-materiellen Entwicklung durch eine bewusste Förderung des sozialen Zusammenhaltes ergänæt werden muss, ist die zentrale Einsicht, die Schmoller aus der historischen Betrachtung von Aufstieg und Zerfall von Nationen und Zivilisationen gewinnt.

Diese konzeptionelle Bestimmung des Verhältnisses von marktwirtschaftlichem Wettbewerb und sozialem Ausgleich findet sich vor allem bei Alfred Müller-Armack wieder, der - neben der Vermittlung durch die Schriften von Max Weber und Werner Sombart - auch unmittelbar mit den Ideen der deutschen historischen Schule um Schmoller vertraut war (vgl. Müller-Armack 1974d: 246f.; vgl. auch Koslowski 1998: 74f.). Entscheidend für den hier interessierenden Zusammenhang ist die Gegenüberstellung von wirtschaftlicher Freiheit und sozialer Gerechtigkeit, die sich bei MüllerArmack finden lässt (vgl. Müller-Armack 1974a: 74f.). Für ihn befinden sich beide

4 Vgl. dazu: Ebner 2006; Goldschmidt/Wohlgemuth 2008.

$5 \quad$ Siehe dazu auch den sogenannten ersten oder ,älteren` Methodenstreit im Verein für Socialpolitik zwischen Schmoller und Carl Menger (vgl. Wallgärtner 1991: 122-142). 
Ideale in einem antagonistischen oder zumindest nicht-harmonischen Verhältnis und müssen durch Gesellschaftspolitik erst miteinander versöhnt werden (vgl. MüllerArmack 1976: 233ff.). Zudem betrachtet Müller-Armack die Marktwirtschaft selbst als moralisch neutralen, technischen Koordinationsapparat, der an dem Wertfundament der Gesellschaft eher zehrt, als es anreichern oder gar ersetzen zu können (vgl. MüllerArmack 1974c: 150). Die Marktwirtschaft ist insofern auf vorgängige Wertorientierungen und gesellschaftspolitische Zielsetzungen angewiesen:

„Was wir verlangen, ist eine neu zu gestaltende Wirtschaftsordnung. Eine solche $[\ldots]$ bedarf der tieferen Begründung durch sittliche Ideale, welche ihr erst die innere Berechtigung verleihen. Zwei grossen sittlichen Zielen fühlen wir uns verpflichtet, der Freibeit und der sozialen Gerechtigkeit. [...] Es würde [..] keine Wirtschaftsordnung heute zu Recht bestehen, wenn sie nur an die Sicherung der Freiheit dächte. Blosse Freiheit könnte zum leeren Begriff werden, wenn sie sich nicht mit der sozialen Gerechtigkeit als verpflichtender Aufgabe verbände. So muss die soziale Gerechtigkeit mit und neben der Freiheit zum integrierenden Bestandteil unserer künftigen Wirtschaftsordnung erhoben werden" (MüllerArmack 1974b: 90, Hervorhebung im Original).

In dieser Hinsicht unterscheiden sich die Ansichten von Müller-Armack wesentlich von den Ansichten der Freiburger Schule innerhalb des Ordoliberalismus (vgl. Goldschmidt/Wohlgemuth 2008: 270).

Für Eucken und Böhm waren Marktwirtschaft und sozialer Ausgleich insofern unmittelbar miteinander verbunden, als allein die marktwirtschaftliche Koordination wirtschaftlicher Freiheit die materielle Basis für eine Verbesserung der allgemeinen sozialen Lage bereitstellt (vgl. Eucken 1952: 312f.). Zudem wurde die marktwirtschaftliche Wettbewerbsordnung von der Freiburger Schule mit einer ethischen Dimension aufgeladen. Diese Dimension wurde vor allem in der Garantie von Freiheit und Gleichheit vor dem Gesetz sowie der Abschaffung von Privilegien gesehen. Unter diesem Blickwinkel ist der Wettbewerb alles andere als ein ethisch neutraler Koordinationsmechanismus. Vielmehr garantiert er die permanente Infragestellung wirtschaftlicher Macht und bildet damit zugleich ein Instrument zur Herstellung individueller Freiheit und sozialer Aufstiegsmöglichkeiten.

„Anders als in Freiheit kann die soziale Frage nicht gelöst werden. [...] In den richtigen Marktformen ist auch die Entartung der Freiheit zur Willkürherrschaft einzelner über viele andere unmöglich. [...] Gerade soziale Gründe zwingen dazu, diese Linie der Wettbewerbsordnung zu verfolgen“ (Eucken 1948: 130f.).

Gerade in diesem Punkte bestand - abgesehen von der unterschiedlichen methodologischen Ausrichtung - Einigkeit zwischen der Freiburger Schule und Ludwig Erhard (vgl. Goldschmidt/Wohlgemuth 2008: 266ff.).

Die politisch wirksame Leitidee der Sozialen Marktwirtschaft stellt letztendlich einen programmatischen Kompromiss zwischen diesen und anderen gedanklichen Strömungen dar. Je nach Gewichtung der unterschiedlichen Facetten innerhalb der Gesamtidee enthält diese unterschiedliche Implikationen für eine konkrete Sozialpolitik. Diese politische Offenheit der Leitidee ist zum einen von ihren politischen Grün- 
dungsvätern explizit intendiert worden ${ }^{6}$ und kann als ein Grund für ihre gesellschaftliche Integrationskraft in der alten Bundesrepublik angesehen werden. Andererseits liegt diese Offenheit in einer konzeptionellen Schwäche begründet: Die an der Entstehung der politischen Leitidee beteiligten konzeptionellen Strömungen müssen bis heute um die Interpretationshoheit über ihr gemeinsames Erbe ringen (vgl. Schlecht 1999: 289), weil keine dieser Strömungen die Argumente der jeweils entgegengesetzten Position überzeugend entkräften konnte.

Auch wenn der Ordoliberalismus für sich beanspruchen darf, einen unmittelbaren Zusammenhang zwischen der Idee des Sozialen und dem marktwirtschaftlichen Wettbewerb dadurch herzustellen, dass er den Wettbewerb über die Idee des Sozialen zu rechtfertigen versucht, so bleibt dennoch unklar, woher eine solche Idee des Sozialen ihrerseits neben dem Prinzip individueller ökonomischer Freiheit ihre Berechtigung erhält. Wäre der Zusammenhang zwischen der Idee des Sozialen und dem Wettbewerbsprinzip tatsächlich vollständig, müsste der Ordoliberalismus auch die Konsequenz eines reinen Marktliberalismus zu tragen bereit sein, der die Idee des Sozialen vollständig in der unsichtbaren Hand des Marktes aufgehen lässt. Das Soziale wäre vollständig eine Funktion individueller Freiheit und könnte damit gerade nicht mehr als eigenständige Basis einer Rechtfertigung von ökonomischer Freiheit und Wettbewerb dienen. Unter solchen konzeptionellen Voraussetzungen würde die Soziale Marktwirtschaft - abgesehen von der Betonung der Bedeutung staatlicher Regelsetzung - zu einer reinen Marktwirtschaft tendieren.

Wenn andererseits im Anschluss an die Tradition der deutschen historischen Schule insbesondere von Müller-Armack die Idee des Sozialen derart verselbständigt wird, dass sie eine weitgehende konzeptionelle und normative Unabhängigkeit gegenüber ökonomischer Freiheit und Wettbewerb beanspruchen darf, dann bleiben die zentralen Koordinationsmechanismen des Kapitalismus einem fortwährenden ethischen Vorbehalt ausgesetzt. Und in der Folge eines derartigen Vorbehaltes müsste immer wieder neu mit dem Aufbrechen der Systemfrage gerechnet werden, so wie sie momentan von Böckenförde gestellt wird.

Weder die faktisch wirksame Leitidee selbst noch ihre theoretisch-konzeptionellen Grundlagen vermögen die Frage zu klären, worin der postulierte Zusammenhang zwischen der Sphäre des Ökonomischen - im Sinne von eigeninteressierten Tauschhandlungen - und der Sphäre des Sozialen - im Sinne einer über das ökonomische Nutzenkalkül hinausgehenden Orientierung ${ }^{7}$ - seine argumentativ zwingende Basis findet. Und nur auf Grundlage einer solchen Basis lässt sich die bis heute virulente Grundsatzfrage klären, ob dem Sozialen innerhalb der Sozialen Marktwirtschaft lediglich die Funktion einer nachträglichen Korrektur mancher Ergebnisse des Wirtschaftsprozesse zukommen soll oder ob das Soziale zusammen mit dem Ökonomischen als eine ursprüngliche konzeptionelle Einheit anzusehen ist. Im ersten Falle bedürfte zumindest der von einer idealen Wettbewerbsordnung gelenkte Wirtschaftsprozess keiner weiteren ethischen Rechtfertigung, Begrenzung oder sozialen Erweiterung. Im zweiten Falle wäre das Ökonomische konstitutiv - faktisch wie auch norma-

6 Vgl. den ,irenischen Ordnungsgedanken“ bei Müller-Armack 1974c: 150.

$7 \quad$ Vgl. die definitorische Abgrenzung zwischen dem Ökonomischen und dem Sozialen in Kapitel 3.2. 
tiv - auf eine soziale Einbettung angewiesen. Selbst der von einer - im Sinne des Ordoliberalismus - ,idealen' Wirtschaftsordnung gelenkte Wirtschaftsprozess könnte den Anforderungen des Sozialen nicht genügen. Es gäbe über die Ergebnisse des idealen Wirtschaftsprozesses hinaus berechtigte gesellschaftliche Belange, die sich einer ökonomischen Sichtweise prinzipiell verschlössen.

\section{Die Rechtfertigung der Sozialen Marktwirtschaft durch den Nachweis des inneren Zusammenhangs zwischen Marktwirtschaft und Sozialer Marktwirtschaft}

\subsection{Aufgabenstellung: Etablierung eines zwingenden Zusammenhangs zwi- schen dem Sozialen und dem Ö̉konomischen}

In den oben angedeuteten Argumentationssträngen bleibt die Idee der Sozialen Marktwirtschaft deshalb unklar und damit anfechtbar, weil die jeweiligen Verbindungslinien, die zwischen dem Ökonomischen und dem Sozialen gezogen werden, allenfalls eine synthesis post factum darstellen. Sowohl das Element des Sozialen als auch das Element des Ökonomischen werden jeweils vorausgesetzt, um anschließend eine Verbindung zwischen beiden Elementen zu konstruieren. Die dargestellten Traditionsstränge unterscheiden sich dabei lediglich in der Frage, von welchem der beiden Elemente sie primär ausgehen, um eine solche Verbindung zu konstruieren. Schematisch vereinfachend lassen sich die beiden Argumentationslinien folgendermaßen veranschaulichen:

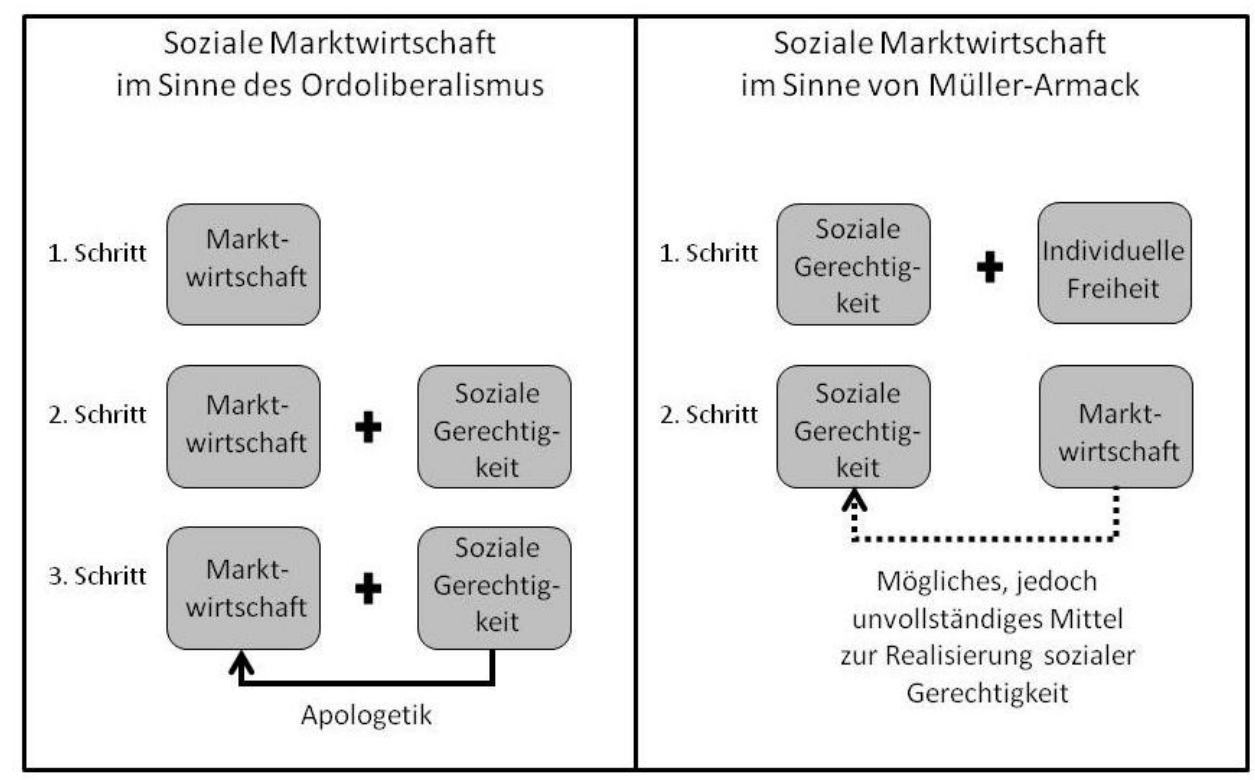

Abbildung 1: Formale Bestimmung des Zusammenhangs zwischen dem Sozialen und dem Ökonomischen (eigene Quelle). 
Im Falle der Interpretation der Sozialen Marktwirtschaft im Sinne des Ordoliberalismus der Freiburger Schule wird die Idee des Sozialen verwendet, um eine nachträgliche Apologie für das Ökonomische zu konstruieren, das jedoch auch ohne diese Apologie normativ Bestand hat. Im Falle der Interpretation der Sozialen Marktwirtschaft im Sinne von Alfred Müller-Armack erscheint das Ökonomische als ein mögliches Instrument zur Realisierung einer Idee des Sozialen. Es erhält damit einen rein hypothetischen Status.

Sollte dem Verhältnis zwischen beiden Elementen eine zwingende Bedeutung gegeben werden können, so müsste zweierlei gezeigt werden. Erstens müsste nachgewiesen werden, dass beide Elemente nicht jeweils einseitig aufeinander zurückgeführt werden können, sondern ihre relative Eigenständigkeit behalten. Ließe sich beispielsweise das Soziale einseitig auf das Ökonomische zurückführen, so wäre zwar ein logisch zwingender Zusammenhang aufgewiesen, jedoch würde das Soziale seine Eigenständigkeit verlieren; die Idee der Sozialen Marktwirtschaft müsste im Sinne des klassischen Liberalismus revidiert werden. Zweitens dürfte ihre Eigenständigkeit nur als eine ausschließlich relative Bestand haben: Weder das Soziale noch das Ökonomische dürften außerhalb ihrer Bezogenheit aufeinander denkbar sein. Beide Begriffe müssten als reine Wechselbestimmungen konzipiert werden. ${ }^{8}$

Mit diesen Bemerkungen werden allein formal-logische Bedingungen angegeben, die eine gedanklich zwingende Rechtfertigung der Sozialen Marktwirtschaft erfüllen müsste. In den folgenden Abschnitten wird es darum gehen, diese formal-logischen Bedingungen auf einer inhaltlichen Ebene einzulösen.

\subsection{Definition des sozialen und des ökonomischen Elementes der Sozialen Marktwirtschaft}

Um den folgenden Versuch einer Verhältnisbestimmung zwischen dem Sozialen und dem Ökonomischen unternehmen zu können, müssen zuvor beide Elemente definiert werden. Im Rahmen dieses Beitrages ist es nicht möglich, diese Definition inhaltlich zu rechtfertigen und ihre eventuelle apriorische Notwendigkeit aufzuzeigen. Da es hier allein um eine Rechtfertigung der Sozialen Marktwirtschaft zu tun ist, sollen beide Begriffe lediglich so aufgenommen werden, wie sie innerhalb dieses Konzeptes verwendet werden:

Unter dem Ökonomischen soll im Folgenden die Perspektive eigeninteressierter Individuen verstanden werden, die in gegenseitiger formaler Freiheit Tauschakte initiieren. Die sich im Tauschakt gegenübertretenden Individuen sollen als formal frei angesehen werden, wenn sie jeweils keinerlei direkten oder indirekten Zwang aufeinander ausüben. Sie schränken ihre ansonsten vollständige Eigeninteressiertheit also in einer einzigen Hinsicht ein: Sie respektieren zumindest faktisch die Entscheidungsfreiheit ihres Gegenübers auch dann, wenn es in ihrem Interesse läge, diese Entscheidungsfreiheit zu missachten. Diese Einschränkung unterscheidet das ökonomische Tauschverhältnis von dem im hypothetischen Naturzustand veranschlagten „Krieg aller gegen alle“ (vgl. Hobbes 1984: 96). 
Unter dem Sozialen soll im Folgenden eine Perspektive verstanden werden, die jedem Individuum als solchem einen absoluten Wert zumisst. Diese Perspektive geht über die ökonomische Perspektive hinaus, weil sie nicht nur die Entscheidungsfreiheit der Individuen in einer jeweils als gegeben unterstellen Entscheidungssituation respektiert, sondern die Bedingungen, unter denen Individuen ihre Entscheidungen treffen, nochmals am Maßstab des absoluten Wertes jedes Individuums beurteilt. Diese Perspektive schließt also nicht nur direkten oder indirekten Zwang zwischen Individuen aus, sondern auch denjenigen Zwang, der von bestimmten Entscheidungssituationen ausgeht. Unter dieser Perspektive wäre z.B. die Bereitschaft eines Menschen, eine seiner beiden Nieren gegen Geld zu verkaufen, nur dann auch materialiter als freiwillig $\mathrm{zu}$ verstehen, wenn die Lebensbedingungen dieses Menschen ihn nicht faktisch dazu nötigen, einen Teil seines Körpers zu verkaufen. Aus ökonomischer Perspektive hingegen wäre gegen die möglichst unreglementierte Etablierung eines solchen Marktes für menschliche Organe gerade auch im Verhältnis zwischen Entwicklungs- und Industrieländern nichts einzuwenden: Denn unter den als gegeben unterstellten Entscheidungsbedingungen kommt der Anbieter der Niere offenbar aufgrund freier Abwägung zu dem Schluss, dass ihn ein Verkauf der Niere besser stellt als ihr Verbleib in seinem Körper. ${ }^{9}$ Die faktische Befähigung des einzelnen Individuums, gemäß einer solchen Abwägung zu handeln, verstehen auch die Väter der Sozialen Marktwirtschaft als Ausdruck des Prinzips ökonomischer Freiheit (vgl. Schlecht 1999: 290f.).

Aus ökonomischer Perspektive ist die Freiheit des Individuums eine Entscheidungsfreiheit ceteris paribus. Damit wird diese Freiheit nicht in ihren Konstitutionsvoraussetzungen betrachtet, sondern vielmehr als in jeder beliebigen Situation tatsächlich bestehend vorausgesetzt (vgl. Gertenbach 2007: 128ff.). Aus diesem Blickwinkel erklärt sich auch die regelmäßige Betonung der ursprünglichen „Natürlichkeit“ der Marktordnung bei Adam Smith und im klassischen Liberalismus (vgl. Ulrich 2001: 170ff.): Wenn Individuen erstens als in Entscheidungsfreiheit existierend und zweitens als sich in dieser Freiheit faktisch respektierend vorausgesetzt werden, dann muss wenn keine weiteren normativen Einschränkungen vorgenommen werden - ihre Interaktion von selbst die Form des marktlichen Austausches annehmen. Insofern stellt dieser die „natürlichste“ Interaktionsform dar. Entscheidend ist dabei die Annahme, dass die Entscheidungsfreiheit - und damit die Rationalität im Sinne der Nutzenmaximierung - des Individuums realistisch als erstes Datum unterstellt wird.

Das Element des Sozialen stellt demgegenüber den Versuch dar, die Konstitutionsvoraussetzungen individueller Freiheit zu reflektieren. Damit wird die faktisch bestehende individuelle Freiheit nicht als Faktum betrachtet, sondern in ihrer Abhängigkeit von den objektiven Gegebenheiten, unter denen sie aktualisiert wird. Der Aufweis der logischen Notwendigkeit dieser rekursiven Erweiterung der Bedeutungsdimension individueller Freiheit ist das zentrale Problem einer wirtschaftsethischen Rechtfertigung der Grundlagen der Sozialen Marktwirtschaft. 


\subsection{Problemdurchführung: Gegenseitiges Anerkennungsverhältnis als Be- dingung der Möglichkeit einer Mehrzahl von Individuen}

Das Element des Sozialen kann in der Bedeutung, in der es zuvor definiert wurde, seine eigene Rationalität gegenüber der scheinbar einfacheren und damit klareren $\mathrm{Be}$ deutung des Ökonomischen von sich aus nicht rechtfertigen. Es erscheint gegenüber dem Ökonomischen lediglich als Ausdruck eines Werturteils und damit als angreifbar. ${ }^{10}$ Soll diese Werturteilsbezogenheit gegenüber dem Ökonomischen als nichtwillkürliche und damit rationale gerechtfertigt werden, muss eine Reflexion auf die Bedingung der Möglichkeit des Ökonomischen erfolgen.

Die ökonomische Perspektive betrachtet einerseits die Welt aus der Perspektive des eigeninteressierten Individuums, andererseits kommt in dieser Welt immer schon eine Mehrzahl von Individuen vor, die miteinander in Beziehung stehen. Der homo oeconomicus ist keine Monade, die in vollständiger gedanklicher Isolation lebt. Die ökonomische Perspektive setzt die Entscheidungsfreiheit des Individuums, nicht eines Individuums voraus. „homo oeconomicus“ bezeichnet kein quantitatives Singulum sondern ist ein hypothetischer Allgemeinbegriff. Sofern die Entscheidungsfreiheit aber explizit als allgemeine gedacht wird, ist damit auch schon die Voraussetzung einer Mehrzahl von Individuen gegeben, die in ihren Handlungen durch die Außenwelt unmittelbar aufeinander bezogen sind.

Es stellt sich jedoch die Frage, welche gedanklichen Voraussetzungen das Ökonomische eingegangen ist, wenn es eine derartige Perspektive konstruiert. Wie lässt sich eine Perspektive konstruieren, die sich selbst als methodischen Individualismus bezeichnet, dabei aber immer schon eine Mehrzahl von Individuen und ihre gegenseitige Bezogenheit im Blick hat? Wenn wir die Perspektive des einzelnen Individuums als Perspektive der Weltbetrachtung wählen und gleichzeitig unterstellen, dieses Individuum weiß um das Vorhandensein anderer Individuen, die es zumindest als mögliche Transaktionspartner akzeptiert, müssen wir uns fragen, welche gedanklichen Operationen das einzelne Individuum zu diesem Wissen befähigen: Wie kann es sich als eigeninteressiertes Individuum verstehen und zugleich von anderen abgrenzen, indem es deren individuelle Entscheidungsfreiheit akzeptiert?11

Mit dieser Frage ist das grundlegende Problem des Zusammenhangs von Individualität und Kollektivität unter der Voraussetzung von Freiheit angesprochen, das insbesondere in der Rechtsphilosophie behandelt wird. Nach Kant ist

„[d]as Recht [...] der Inbegriff der Bedingungen, unter denen die Willkür des einen mit der Willkür des andern nach einem allgemeinen Gesetze der Freiheit zusammen vereinigt werden kann" (Kant 1797: 230).

Die entscheidend weitergehende Frage wurde in der Rechtsphilosophie von Johann Gottlieb Fichte gestellt: Wie können sich Menschen, die sich als frei verstehen, über-

10 Zur Bedeutung und zur Reichweite von „Werturteilen“ in der Wissenschaft vgl. Weber 1973: insbes. $500 \mathrm{ff}$.

11 Die Bedeutung dieser Frage für die ökonomische Wissenschaft liegt in dem grundlegenden Problem, wie der Übergang von einer singulären Eigeninteressiertheit zu der aggregierten Perspektive der Gesamtheit eigeninteressierter Individuen konstruiert werden kann. Wie die spieltheoretischen Modellierungen zeigen, konvergieren beide Perspektiven keinesfalls zwangsläufig. 
haupt voneinander unterscheiden? Diese Frage zielt ab auf die Bedingungen der Möglichkeit der Behauptung einer Mehrzahl von Individuen und damit der epistemologischen Bedingungen des Zusammenlebens von Menschen. ${ }^{12}$

Diese Bedingungen der Möglichkeit können nur innerhalb einer voraussetzungsreichen transzendentalphilosophischen Rekonstruktion der Bedingungen des Bewusstseins und damit zugleich der Bedingungen der Welterfahrung entwickelt werden. Eine solche Rekonstruktion sprengt den Rahmen dieses Beitrages. ${ }^{13}$ Gleichwohl soll hier der zentrale Gedanke einer solchen Rekonstruktion bezogen auf das Problem der Abgrenzung individueller Entscheidungsfreiheiten zumindest dargestellt werden.

Sofern Freiheit überhaupt veranschlagt werden soll, muss jede Einschränkung von Freiheit gerechtfertigt werden. Freiheit als gedankliches Postulat ist, sofern überhaupt von Freiheit die Rede sein soll, zunächst als unbegrenzt, als absolut zu veranschlagen. ${ }^{14}$ Jede Qualifizierung von Freiheit muss gegenüber diesem Ursprungspostulat in ihrer gedanklichen Notwendigkeit gerechtfertigt werden. Wenn ein Individuum von der Möglichkeit anderer Individuen weiß, dann stellt dieses Wissen bereits eine entscheidende Einschränkung seiner eigenen Freiheit dar: Seine Freiheit findet allein faktisch eine absolute Grenze in den Freiheitsentscheidungen eines möglichen Gegenübers. Dieses Gegenüber ist nicht nur in dem Sinne widerständig, in dem die reale Außenwelt nicht unmittelbar dem menschlichen Willen unterliegt. Vielmehr tritt eine andere Freiheit auf, die allein in ihrer Existenz dem Freiheitswillen des betrachteten Ausgangsindividuums eine absolute Grenze gegenüberstellt.

Die Gedanken der absoluten, unbeschränkten Freiheit einerseits und der ebenso absoluten Beschränkung dieser Freiheit andererseits schließen sich gegenseitig aus: Keiner dieser beiden Gedanken kann einen Richtigkeitsanspruch erheben, ohne zugleich den des anderen zu negieren. Dennoch sind beide Gedanken in der Behauptung einer Mehrzahl von Individuen, die sich jeweils als frei verstehen, enthalten. Soll die Verstehbarkeit dieser Behauptung - deren faktische Plausibilität nicht in Frage zu stellen ist - philosophisch rekonstruiert werden, so müssen die sich gegenseitig ausschließenden Gedankeninhalte - unbeschränkte Freiheit, Beschränkung - in einer gedanklichen Leistung vereinigt, synthetisiert werden. Diese gedankliche Leistung stellt also eine verstehbare Verbindung zwischen beiden Gedanken her, die ihren jeweiligen Gültigkeitsanspruch dennoch behalten.

Soll unbeschränkte Freiheit als beschränkt gedacht werden können, so scheint das offenbar nur im Rahmen einer freiwilligen Einschränkung geschehen zu können. Würde die Freiwilligkeit der Einschränkung jedoch bereits als vollständige Antwort auf das Ausgangsproblem angesehen, würde schlicht derjenige gedankliche Gehalt vorausgesetzt, der ja erst rekonstruiert werden sollte: Die Möglichkeit der Einschrän-

12 Fichte hat den Gedanken der apriorischen, vor-individuellen Konstituierung von Individualität ausführlich im Ersten Hauptstück „Deduktion des Begriffs vom Rechte“ der „Grundlage des Naturrechts" behandelt. Vgl. Fichte 1796: 329-360.

13 Für eine genauere Entwicklung der transzendentalphilosophischen Rekonstruktion des Rechtes vgl. Trautnitz 2008: 277ff.

14 Vgl. Fichtes Bestimmung der „Tathandlung“ (Fichte 1794/95: 255ff.) sowie die entsprechende Interpretation bei Janke 1990: 103. 
kung einer unbeschränkten Freiheit! Die gesuchte Lösung muss deshalb neben der Freiwilligkeit ein weiteres Element enthalten. Es müsste verständlich werden, inwiefern die veranschlagte Freiwilligkeit der Einschränkung ihrerseits überhaupt erst zu dieser Freiwilligkeit instand gesetzt wird. Hier ist also insbesondere keine solitäre Leistung eines bereits als existierend gedachten Individuums zu veranschlagen, das sich selbst in bestimmter Hinsicht freiwillig einschränkt.

Die Beschränkung unbeschränkter Freiheit muss also im Rahmen einer solchen gedanklichen Leistung entstehen, die die Ermöglichungsbedingung zu dieser Freiwilligkeit der Beschränkung zugleich enthält. Soll die gesuchte Lösung in einem Gedanken bestehen, darf die Ermöglichungsbedingung jedoch nicht in eine weitere Instanz ausgelagert werden. Sie muss vielmehr innerhalb der zu suchenden Lösung generiert werden: Sowohl die Ermöglichungsbedingung der Freiwilligkeit als auch die tatsächliche freiwillige Beschränkung sollen in ein und demselben Gedanken enthalten sein.

Aus dem Gesagten folgt die Notwendigkeit einer geschlossenen Gegenseitigkeit: Die notwendige aber nicht hinreichende Freiwilligkeit der Beschränkung muss zu dieser Freiwilligkeit instand gesetzt werden (Ermöglichungsbedingung), und diese Ermöglichungsbedingung darf von nichts anderem abhängen als von der Freiwilligkeit. Damit würde die gesuchte Lösung in einem geschlossenen Verhältnis (zwischen zwei Polen) bestehen, welches die Pole jeweils für sich in die Lage versetzt, das Verhältnis zu generieren. Die gesuchte Lösung muss also offenbar in einer solchen freiwilligen Einschränkung von Freiheit bestehen, die erstens ihrerseits von einer anderen freiwilligen Einschränkung ermöglicht wird und zweitens zugleich diese andere freiwillige Einschränkung ermöglicht. Damit liegt die Lösung des Ausgangsproblems offenbar in einer apriorischen (vorindividuellen), gegenseitig verschränkten Anerkennungsleistung. ${ }^{15}$ Diese apriorische Anerkennungsleistung lässt sich allein über ihr faktisches Ergebnis veranschaulichen: Nämlich durch die Möglichkeit einer a posteriori vollzogenen, gegenseitigen freien Anerkennung von Individuen als frei. Als Ergebnis dieser Überlegungen ist festzuhalten, dass die Behauptung einer Mehrzahl von Individuen auf Grundlage des methodischen Individualismus nur möglich ist, sofern dem Individuum das Wissen um die Möglichkeit eines Anderen zugeschrieben wird. Dieses Wissen setzt jedoch den Gedanken einer gegenseitigen, freiwilligen Anerkennung und damit Einschränkung von Freiheit durch Freiheit voraus:

„Das Verhältniß freier Wesen zu einander ist demnach nothwendig auf folgende Weise bestimmt, und wird gesezt, als so bestimmt: Die Erkenntniß des Einen Individuums vom anderen, ist bedingt dadurch, daß das andere es als ein freies

Die apriorische Dimension dieser Anerkennungsleistung muss veranschlagt werden, um dem Fehler des realistischen Zirkelschlusses zu entgehen. Diese Dimension ist innerhalb des transzendentalphilosophischen Ansatzes gerade nicht als metaphysische Wirklichkeit zu substantialisieren, sondern lediglich als notwendiges Postulat zu verstehen: Es soll damit allein eine logisch unvermeidbare Negation bezeichnet werden: Denke die Anerkennung nicht als willkürliche Leistung von Individuen! Gleichwohl hat die Betonung der apriorischen Dimension der Anerkennung erhebliche Implikationen für das Verhältnis zwischen Rechtsidee und positivem Recht. Dieses wird damit an eine Legitimitätsbedingung zurückgebunden, die ihm selbst nicht verfügbar ist. Zur apriorischen Bedeutung des Rechts vgl. Janke 1990: 102. 
behandle, (d. i. seine Freiheit beschränke durch den Begriff der Freiheit des ersten.) Diese Weise der Behandlung aber ist bedingt, durch die Handelsweise des ersten gegen das andere; diese durch die Handelsweise, und durch die Erkenntniß des anderen, und so ins unendliche fort. Das Verhältniß freier Wesen zu einander ist daher das Verhältniß einer Wechselwirkung durch Intelligenz und Freiheit. Keines kann das andere anerkennen, wenn nicht beide sich gegenseitig anerkennen: und keines kann das andere behandeln als ein freies Wesen, wenn nicht beide sich gegenseitig so behandeln“ (Fichte 1796: 351).

Die Reflexion auf die Bedingungen der Möglichkeit von Individualität führt damit zu dem Nachweis, dass sich die realistisch unbestreitbare Tatsache der Existenz von freien und damit auch gegeneinander abgegrenzten Individuen gedanklich nur durch die Annahme einer apriorischen Anerkennungsleistung rekonstruieren lässt. Für die hier verfolgten Zwecke ist vor allem eine Implikation dieser Rekonstruktion entscheidend, die auch in obigem Zitat angesprochen wird: Der Nachweis der Abhängigkeit individueller Freiheit von einer gegenseitigen Anerkennung, die dem einzelnen Individuum gerade nicht zur Disposition steht. Dieser Nachweis durchbricht die Realistik der These von der Entscheidungsfreiheit des einzelnen Individuums, die den Kern des Ökonomischen ausmacht. Vielmehr zeigt dieser Nachweis die normative Bedeutung der Entscheidungsfreiheit des Individuums: Individuen können sich als frei nur verstehen, sofern sie sich als sich gegenseitig anerkennend begreifen. ${ }^{16}$ Diese Anerkennung weist damit einerseits über das einzelne Individuum hinaus auf einen Ursprung, der dem einzelnen Individuum nicht mehr verfügbar ist und in diesem spezifischen Sinne als ,kollektiv` bezeichnet werden kann. Andererseits wird diese Individualität aber gerade anerkannt, und damit sowohl faktisch gesetzt als auch vollständig legitimiert.

\subsection{Der innere Zusammenhang zwischen Marktwirtschaft und Sozialer Marktwirtschaft}

Individuelle Freiheit konstituiert sich in einem prädeliberativen Anerkennungsverhältnis. Dieses Anerkennungsverhältnis entlässt aus sich die Facta bestehender Freiheitssphären - in der rudimentärsten Form: die formale Entscheidungsfreiheit einer Mehrzahl von Individuen. Sobald diese Individuen faktisch aufeinandertreffen, entsteht das Problem der Koordinierung ihrer Freiheitsentscheidungen. Dieses pragmatische Problem stellt jedoch aus transzendentalphilosophischer Perspektive lediglich eine Aktualisierung der apriorischen Anerkennungsproblematik dar: Sofern Individuen sich in einem Tauschakt als formal frei anerkennen, folgen sie einer normativen Festlegung, der sie ihre formale Entscheidungsfreiheit überhaupt erst verdanken, nämlich der gegenseitigen Anerkennung und damit Abgrenzung von Freiheitssphären.

Sofern das Soziale in dem zuvor definierten Sinne die Konstitutionsbedingungen individueller Freiheit auf einer faktischen Ebene reflektiert, ist es als die empirische Konkretisierung des apriorischen Anerkennungsverhältnisses zu verstehen: Insofern kein Individuum seine formale Freiheit sich selbst verdankt, jedes Individuum aber Anspruch auf diese formale Freiheit in gleichem Maße hat, verbietet es sich, die faktisch

16 Es ist hier nochmals zu betonen, dass sich der transzendentalphilosophische Ansatz auf der Ebene epistemischer Bedingungen und nicht auf der Ebene realistischer Handlungsweisen bewegt. 
bestehende Verteilung an individuellen Entfaltungsmöglichkeiten unter Berufung auf den Wert individueller Freiheit für prinzipiell sakrosankt zu erklären. Das apriorische Anerkennungsverhältnis verweist auf die Möglichkeit der Kritik der faktischen Lebensbedingungen von Menschen, insofern es die individuelle Freiheit entnaturalisiert und in ihren Entstehungsbedingungen reflektiert. Das Soziale der Sozialen Marktwirtschaft ist deshalb auch nicht zu denken als mehr oder weniger beliebige Erweiterung der „natürlichen“, kraft faktischer Funktionsfähigkeit legitimierten Marktwirtschaft. Das Soziale zeigt sich vielmehr als die Vervollständigung des marktwirtschaftlichen Elementes im Hinblick auf dessen Entstehungszusammenhang. Sofern individuelle Freiheit als Systemwert des Kapitalismus veranschlagt wird, zeigen sich das Soziale wie das Ökonomische als unmittelbare Entsprechung dieses Wertes.

Die Rechtfertigung des Sozialen als nicht-willkürliche Vervollständigung des Ökonomischen deckt aber zugleich auch die legitime Eigenständigkeit des Ökonomischen auf: Die apriorische Anerkennungsleistung, die als gedankliches Postulat veranschlagt werden muss, sofern die faktische Abgrenzung von Individuen verstanden werden soll, weist die Entscheidungsfreiheit des Individuums als legitime aus. Das Ökonomische ist in seinem Ursprung keine privative Erscheinungsform des Sozialen, als das es von Böckenförde beschrieben wird. Es ist vielmehr als vollständig legitime Erscheinungsform menschlicher Freiheit verstehbar! Individuelle Freiheit zeigt sich notwendig als faktische Entscheidungsfreiheit und ist auf Grundlage des Nachweises ihrer Konstitutionsbedingungen als solche zu respektieren. Mit der Salvierung dieser Entscheidungsfreiheit sind aber notwendig die systemischen Konsequenzen verbunden, die Böckenförde beschreibt: Die utilitaristische Kategorie des Eigennutzes entfaltet eine systembildende Kraft. Dieser Befund ist richtig und das, was er beschreibt, kann auf Grundlage der hier vorgetragenen Argumentation als notwendige Vereinseitigung beschrieben werden. Diese Vereinseitigung ist notwendig, insofern individuelle Entscheidungsfreiheit als faktische wie auch normative Grundlage des ökonomischen Systems als legitim ausgewiesen ist. Die systembildende Kraft des Eigennutzes bleibt aber dennoch insofern eine Vereinseitigung, als sich die faktisch bestehende Individualität einem Anerkennungsverhältnis verdankt, das aus sich ebenso die scheinbare Vereinseitigung des Sozialen entlässt.

Die Beseitigung der konzeptionellen Unschärfe im Konzept der Sozialen Marktwirtschaft rechtfertigt nicht nur die Notwendigkeit des Sozialen sondern damit zugleich und gleichwertig auch die Notwendigkeit des Ökonomischen. Die systemische Spannung zwischen beiden Elementen ist nicht auf einen Konstruktionsfehler einer der beiden Seiten zurückzuführen, sondern - unter der Voraussetzung des absoluten Wertes jedes Menschen - als unvermeidbar zu akzeptieren. Die Diagnose einer unaufhebbaren Spannung zwischen beiden Elementen rechtfertigt damit gerade keinen prinzipiellen Umbau des Kapitalismus, sondern verdeutlicht die Notwendigkeit der Begrenzung seiner systemischen Folgen sowohl für die faktische Entscheidungsfreiheit der Individuen als auch für ihre sozialen Beziehungen außerhalb des Marktes.

\section{Systemwechsel oder soziale Einbettung des Ökonomischen?}

Die Diagnose Böckenfördes hinsichtlich der systembildenden Kraft des Eigennutzes, die dazu führt, dass die formaliter frei handelnden Subjekte zunehmend alle Bereiche 
ihres Lebens unter ökonomischen Aspekten betrachten (müssen) und damit sich selbst und ihr Leben zum Objekt von Anpassungszwängen degradieren, ist nicht neu. Eindringlich hatte bereits 1905 Max Weber diese Kraft beschrieben als

,jenen mächtigen Kosmos der modernen, an die technischen und ökonomischen Voraussetzungen mechanisch-maschineller Produktion gebundenen, Wirtschaftsordnung [...], der heute den Lebensstil aller einzelnen, die in dies Triebwerk hineingeboren werden - nicht nur der direkt ökonomisch Erwerbstätigen -, mit überwältigendem Zwange bestimmt und bestimmen wird, bis der letzte Zentner fossilen Brennstoffs verglüht ist“" (Weber 2000: 153, Hervorhebungen im Original).

Die letzte Finanz- und Wirtschaftskrise hat diesen an sich nicht neuen Befund des systemischen Zwanges jedoch deshalb so beunruhigend werden lassen, weil sie offenbart, dass jede noch so weitsichtige Regulierung des Erwerbsinteresses immer von neuem durch die systemischen Wettbewerbskräfte unterlaufen wird, z. B. indem die Finanzbranche nach Abschluss der gegenwärtigen Regulierungsbemühungen Produkte entwerfen wird (aus Gründen des Wettbewerbs entwerfen muss), die so strukturiert sein werden, dass sie von der dann geltenden Regulierung gerade nicht mehr erfasst werden können, ohne jedoch deshalb schon illegal zu sein.

Diese wettbewerbsinduzierte Tendenz zur Umgehung regulatorischer Vorgaben ist eine der unvermeidlichen systemischen Folgen eines nach seiner immanenten Funktionslogik strukturierten Wirtschaftssystems. Diese auf dem Prinzip individueller Freiheit aufbauende Funktionslogik ist jedoch auf Grundlage der zuvor dargestellten Argumentation als legitimiert zu betrachtet und kann somit auch nicht im Sinne eines Systemwechsels grundsätzlich infrage gestellt werden. Dennoch bleibt kritisch zu bedenken, inwiefern die systemischen Folgen dieser Funktionslogik das sie bedingende Fundament der Genese individueller Freiheit untergraben.

Diese Frage zielt auf die Bedingungen ab, unter denen Individuen ihre Freiheit faktisch ausüben können, also auf den zuvor thematisierten Aspekt des Sozialen. Aus der Perspektive dieser Frage ist der Kapitalismus nicht dadurch zu bändigen, dass ein Eingriff in seine Funktionsmechanismen erfolgt, sondern dadurch, dass sein Anwendungsbereich begrenzt wird: Die Individuen sind von der Rechtsordnung in die Lage zu versetzen, ihre Freiheit jederzeit faktisch ausüben zu können. Sie müssten also in der Lage sein, sich den systemischen Zwängen des Marktes auch verweigern zu können. Aus diesem Gedanken lässt sich nach Auffassung des Autors ein Grundrecht auf Partizipationsverweigerung ableiten (vgl. Trautnitz 2008: 330ff.).

Ein solches Grundrecht würde die marktliche Zwangsdynamik nicht modifizieren, jedoch zugleich sicherstellten, dass die Individuen ein Leben nach ihren individuellen Vorstellungen führen können, auch wenn sie nicht am global vernetzten Marktsystem teilnehmen. Als mögliche Konkretisierung eines solchen Grundrechtes auf Partizipationsverweigerung könnte die Idee eines bedingungslosen Grundeinkommens relevant sein. Jedenfalls können systemische Zusammenhänge, wie sie insbesondere durch die Kategorie des individuellen Erwerbsinteresses geschaffen werden, nicht durch die Adressierung der moralischen Substanz der einzelnen Individuen gestaltet werden, sondern allein durch die unpersönliche Konstruktion von Rechtsansprüchen - insbe- 
sondere in der Sozialgesetzgebung, im Arbeitsrecht, im Umwelt- und Verbraucherschutz.

Die hier nur angedeuteten Überlegungen, wie die Funktion des Sozialen gegenüber den ökonomischen Systemzwängen zur Geltung gebracht werden kann, machen deutlich, dass die Intention Böckenfördes, den ,,inhumanen Charakter“ des Kapitalismus zu überwinden, nur innerhalb der Sozialen Marktwirtschaft umgesetzt werden kann. Die Funktionslogik des Kapitalismus ist weder faktisch noch normativ zu modifizieren, sondern bedarf konstitutiv der sozialen Einbettung.

\section{Literaturverzeichnis}

Böckenförde, E.-W. (2009): Woran der Kapitalismus krankt, in: Süddeutsche Zeitung, Jg. 94, 24.04.2009, 8-9.

Dörre, K./ Lessenich, S./ Rosa, H. (2010): Soziologie - Kapitalismus - Kritik. Eine Debatte, Frankfurt am Main: Suhrkamp.

Ebner, A. (2006): The intellectual foundations of the social market economy. Theory, policy, and implications for European Integration, in: Journal of Economic Studies, Vol. 33/No. 3, 206-223.

Eucken, W. (1948): Die soziale Frage, in: Synopsis - Festgabe für Alfred Weber, Heidelberg: Lambert Schneider, 111-131.

Eucken, W. (1952): Grundsätze der Wirtschaftspolitik, Unter Mitarbeit von Edith Eucken und Hensel, P. K. (Hrsg.). Bern/Tübingen: A. Franke AG. Verlag: Mohr-Siebeck.

Fichte, J. G. (1794/95): Grundlage der gesammten Wissenschaftslehre als Handschrift für seine Zuhörer, zitiert nach: J.G.Fichte-Gesamtausgabe der Bayerischen Akademie der Wissenschaften, hrsg. von Reinhard Lauth und Hans Jacob, 1. Werke, Band 2, hrsg. von Reinhard Lauth und Hans Jacob unter Mitwirkung von Manfred Zahn, S. 173-451, Stuttgart - Bad Cannstatt: Frommann-Holzboog.

Fichte, J. G. (1796): Grundlage des Naturrechts nach Principien der Wissenschaftslehre, zitiert nach: J.G.Fichte-Gesamtausgabe der Bayerischen Akademie der Wissenschaften, hrsg. von Reinhard Lauth und Hans Jacob, 1. Werke, Band 3, hrsg. von Reinhard Lauth und Hans Jacob unter Mitwirkung von Richard Schottky, S. 291-460, Stuttgart - Bad Cannstatt: Frommann-Holzboog.

Gertenbach, L. (2007): Die Kultivierung des Marktes - Foucault und die Gouvernementalität des Neoliberalismus, Berlin: Parodos Verlag.

Goldschmidt, N./ Woblgemuth, M. (2008): Social Market Economy: origins, meanings and interpretations, in: Constitutional Political Economy, Vol. 19/No. 3, 261-276.

Hobbes, T. (1984): Leviathan - Oder Stoff Form und Gewalt eines kirchlichen und bürgerlichen Staates. 1. Aufl. Fetscher, Iring (Hrsg.), Frankfurt am Main: Suhrkamp.

Janke, W. (1990): Anerkennung - Fichtes Grundlegung des Rechtsgrundes, in: Girndt, H. (Hrsg.): Selbstbehauptung und Anerkennung: Spinoza - Kant - Fichte - Hegel, Sankt Augustin: Academia Verlag, 95-117.

Kant, I. (1797): Die Metaphysik der Sitten, zitiert nach: Kants gesammelte Schriften, hrsg. von der Königlich Preußischen Akademie der Wissenschaften, Erste Abteilung, Band 6, Berlin: Reimer, 373-493.

Koslowski, P. (1998): The social market economy: social equilibration of capitalism and consideration of the totality of the economic order. Notes on Alfred Müller-Armack, in: Koslowski, P. (Hrsg.): The Social Market Economy: Theory and Ethics of the Economic Order, Berlin: Springer-Verlag, 73-95. 
Müller-Armack, A. (1974a): Die Wirtschaftsordnung, sozial gesehen, wiederabgedruckt in: Müller-Armack, A.: Genealogie der Sozialen Marktwirtschaft: Frühschriften und weiterführende Konzepte, Bern/Stuttgart: Haupt, 73-89.

Müller-Armack, A. (1974b): Vorschläge zur Verwirklichung der Sozialen Marktwirtschaft, wiederabgedruckt in: Müller-Armack, A.: Genealogie der Sozialen Marktwirtschaft: Frühschriften und weiterführende Konzepte, Bern/Stuttgart: Haupt, 90-107.

Müller-Armack, A. (1974c): Das gesellschaftspolitische Leitbild der Sozialen Marktwirtschaft, wiederabgedruckt in: Müller-Armack, A.: Genealogie der Sozialen Marktwirtschaft: Frühschriften und weiterführende Konzepte, Bern/Stuttgart: Haupt, 146-162.

Müller-Armack, A. (1974d): Die wissenschaftlichen Ursprünge der Sozialen Marktwirtschaft, wiederabgedruckt in: Müller-Armack, A.: Genealogie der Sozialen Marktwirtschaft: Frühschriften und weiterführende Konzepte, Bern/Stuttgart: Haupt, 244-251.

Müller-Armack, A. (1976): Stil und Ordnung der Sozialen Marktwirtschaft (1952). In: MüllerArmack, A. (Hrsg.): Wirtschaftsordnung und Wirtschaftspolitik. Studien und Konzepte zur Sozialen Marktwirtschaft und zur Europäischen Integration. 2. Aufl. Bern/Stuttgart: Paul Haupt, 231-242.

Ptak, R. (2004): Vom Ordoliberalsimus zur Sozialen Marktwirtschaft. Stationen des Neoliberalismus in Deutschland, Opladen: Leske \& Budrich.

Schallmayer, P. (2009): Kaptalismuskritik. Theorie und Praxis bei Marx, Nietzsche, Mann, Müntefering und in der Heuschreckendebatte, Würzburg: Königshausen \& Neumann.

Schlecht, O. (1999): Prinzipien einer sozialen Marktwirtschaft., in: Korff, W. (Hrsg.): Handbuch der Wirtschaftsethik, Bd. 2, Gütersloh: Gütersloher Verlagshaus, 289-303.

Schmoller, Gustav (1898): Die Volkswirtschaft, die Volkswirtschaftslehre und ihre Methode, in: Schmoller, Gustav (Hrsg.): Über einige Grundfragen der Socialpolitik und der Volkswirtschaftslehre, Leipzig: Duncker und Humblot, 213-314.

Schmoller, G. (1901): Grundriß der Allgemeinen Volkswirtschaftslehre, Leipzig: Duncker und Humblot.

Schmoller, G. (1918): Die soziale Frage: Klassenbildung, Arbeiterfrage, Klassenkampf, Leipzig: Duncker und Humblot.

Trautnitz, G. (2008): Normative Grundlagen der Wirtschaftsethik. Ein Beitrag zur Bestimmung ihres Ausgangsparadigmas, Leipzig: Duncker und Humblot.

Trojanow, I. (2009): Reform oder Revolution, in: Die ZEIT, 02.04.2009, Nr. 15.

Wallgärtner, G. (1991): Der soziologische Diskurs im Kaiserreich. Auswertung sozialwissenschaftlicher Zeitschriften; Beiträge zur Geschichte der Soziologie 2, Münster: LIT Verlag.

Weber, M. (1973): Der Sinn der ,Wertfreiheit' der soziologischen und ökonomischen Wissenschaften. 1917, in: Winckelmann J. (Hrsg.): Max Weber - Gesammelte Aufsätze zur Wissenschaftslehre. 4. erneut durchgesehene Auflage, Tübingen: Mohr-Siebeck, 489540.

Weber, M. (2000): Die protestantische Ethik und der ,Geist` des Kapitalismus, Textausgabe auf der Grundlage der ersten Fassung von 1904/05 mit einem Verzeichnis der wichtigsten Zusätze und Veränderungen aus der zweiten Fassung von 1920, hrsg. von Klaus Lichtblau und Johannes Weiß, 3. Aufl., Weinheim: Beltz Athenäum Verlag. 